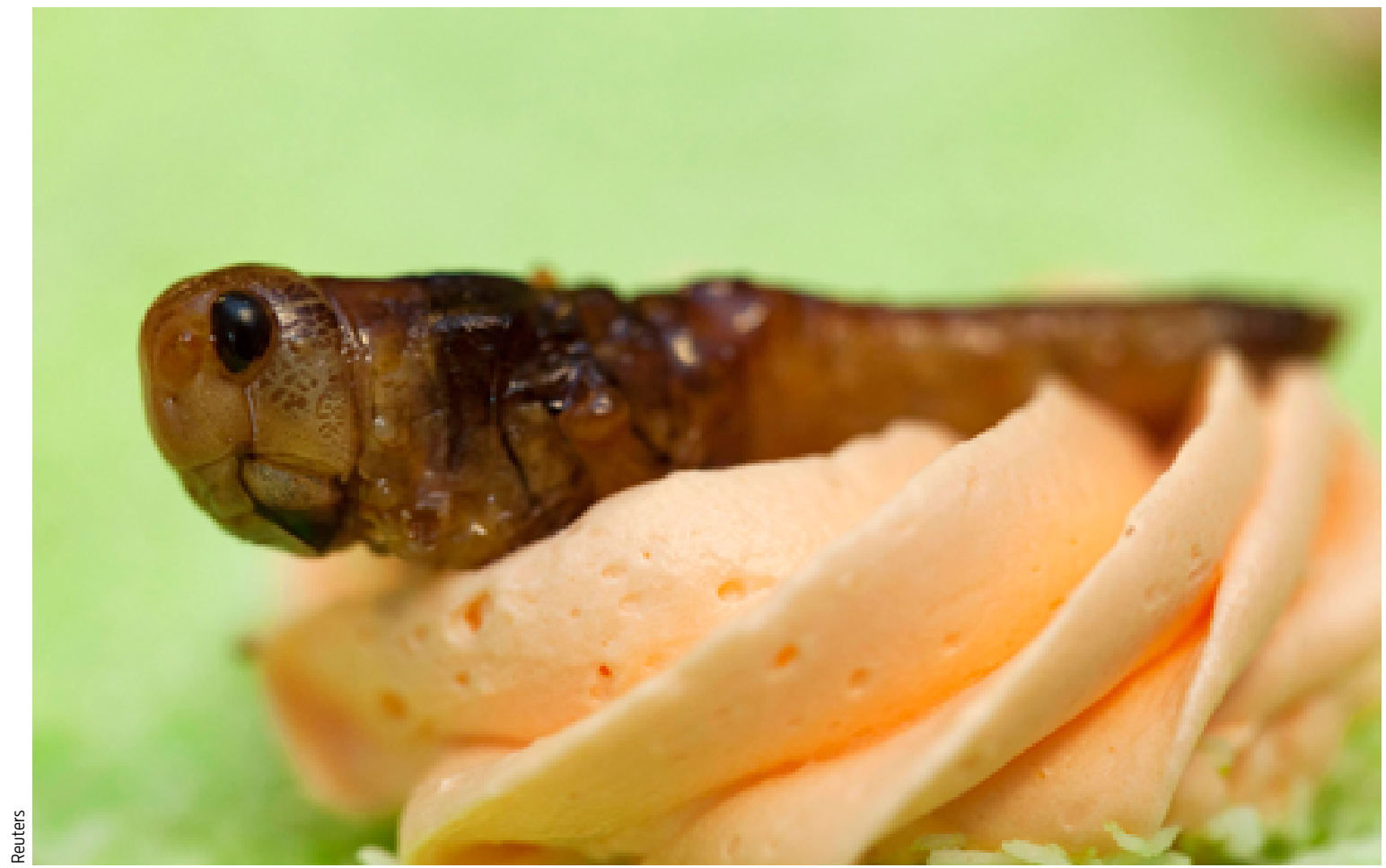

A recipe idea for

MasterChef perhaps?

A caramelised locust

decorates a cake made of insects at the University of Wageningen in the Netherlands. Research at the university showed that insects could provide the best source of protein to meet the food needs of growing populations.

\title{
From The Lowitja Institute
}

\section{Maximising the impact of Aboriginal and Torres Strait Islander health research}

The Lowitja Institute is Australia's National Institute for Aboriginal and Torres Strait Islander Health Research. It is the only research organisation in Australia with a sole focus on the health and wellbeing of Aboriginal and Torres Strait Islander peoples. The institute is governed by a skills-based board, with a majority Aboriginal membership. Currently hosting the Cooperative Research Centre (CRC) for Aboriginal and Torres Strait Islander Health, our collaborative approach to research has developed over three iterations of CRCs since 1997 (Med J Aust 2011; 194: 532-534).

This approach to research is known as the facilitated development approach. It ensures that Aboriginal and Torres Strait Islander peoples and organisations have a strong voice throughout the research process; from setting the agenda, formulating the questions and participating in the research to, most importantly, translating research knowledge into evidencebased policy and practice.

In Darwin on 4-5 June 2012, the Lowitja Institute hosted three roundtable discussions to identify research questions targeting better health outcomes for Aboriginal and Torres Strait Islander peoples. The three roundtables explored nutrition, telehealth, and environmental health and housing research for the Northern Territory. Each prioritised research questions to be further developed. The entire process was participative and involved collaboration between researchers and the end users of research. Of the 44 participants, a third were from Aboriginal organisations, a third from research organisations, and the remainder

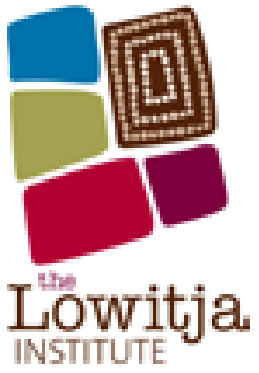

from other non-government organisations and government. We will publish the resulting research priorities at www.lowitja.org.au in July, and will encourage research collaborations to start developing these into grant applications to the National Health and Medical Research Council and other funding bodies.

This is the first time we have used the facilitated development approach to specifically inform external grant applications rather than our own funded research. We want our collaborative development approach to be used more widely. More research with early involvement of research end users should lead to more translation into changed policies and practices that will improve health outcomes for Aboriginal and Torres Strait Islander peoples.
David P Thomas

Associate Director

Lyn Brodie

Chief Executive Officer

The Lowitja Institute

doi: 10.5694/mjal2.10928 


\section{Increased imaging doubles high-dose radiation}

A US study has found a substantial increase in the number of patients exposed to high-dose radiation due to the growing use of advanced diagnostic imaging such as computed tomography (CT) scans. Analysis of imaging data from six large health maintenance organisations showed that the use of CT scans had almost tripled, increasing from 52 per 1000 patients in 1996 to 149 per 1000 patients in 2010. The mean per capita radiation dose had doubled over the same period. Additionally, the proportion of these patients who received a high or very high dose of radiation in a given year had doubled.

The researchers said the increased use of medical imaging had almost certainly contributed to improved patient care and outcomes, but there were few data to quantify the benefits of imaging, and that evidence-based guidelines were needed to "clearly balance benefits against financial costs and health risks". An editorial in the same issue said the study did not show whether the health benefits of the diagnostic information outweighed the radiation risks of imaging, but the data suggested that clinicians should consider - and discuss with their patients - radiation risks when ordering tests, taking into account a patient's cumulative radiation exposure.

The study followed research published in the Lancet last month, which found that children who had repeated CT scans were at higher risk of brain tumours and leukaemia.

JAMA 2012; 13 June (online)

\section{From the MJA archives}

\section{MJA 1992; 20 April (edited extract)}

\section{Aboriginal health: talk but no action}

Equity and social justice were crucial to improving the health and social status of Aboriginal people, the Minister for Aboriginal Affairs, Mr Robert Tickner, told the Public Health Association's 1991 conference on the health of Indigenous people. The government had begun a decade-long process of education and exploration to prepare for a formal process of reconciliation, he said.

Aboriginal speakers said there had been much rhetoric about the need for change and community control but little action, with reports left to gather dust, notably the Royal Commission into Aboriginal Deaths in Custody, which had not been implemented.

Substance abuse was a recurring theme at the conference, with speakers calling for a combined approach of community and legislative controls, and challenging the NT Government's compromised stance on alcohol licensing, which favoured the tourist industry over the needs of the Aboriginal community.

Other papers covered chronic diseases, with one study of 3000

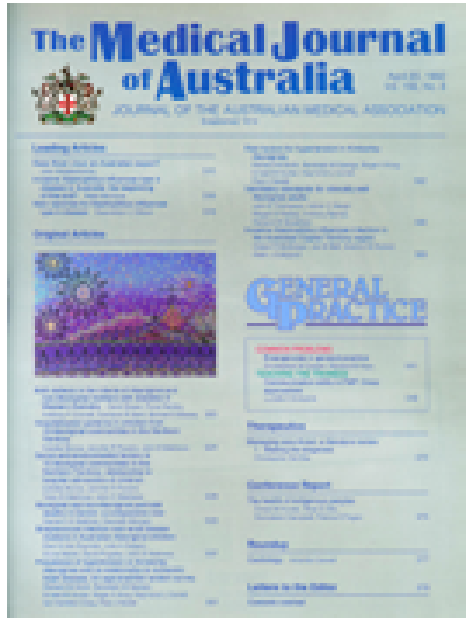

Aboriginal people finding that the rates of end-stage renal disease in some Aboriginal areas were up to 20 times the national average.

One study reported that mental health problems were among the most common clinical problems encountered by doctors in Aboriginal Medical Services, and these could only be solved if social and political factors were addressed.

Anthony Radford, of Flinders University, emphasised the need for social action after reporting the findings of a survey of Aboriginal heads-of-households that examined patterns of self-destructive behaviour.

\section{Surgery promising after genital mutilation}

Reconstruction surgery after female genital mutilation can restore sexual pleasure and ease pain, a French study has found. The study involved almost 3000 women mainly from Mali, Senegal and Ivory Coast — who had clitoral reconstruction after genital mutilation, with 564 having genital mutilation within France. Of the 866 women who were interviewed at 1-year follow-up, almost all (821) said their pain had improved or was no worse, while 815 said clitoral pleasure had improved or was no worse. Around half the women (430) had experienced orgasms. Between 130 and 140 million women worldwide have had genital mutilation in the past 10 years, the researchers said. "Efforts to end this procedure started decades ago, but require major social changes. Repairing the mutilation is an interim solution."

Lancet 2012; 12 June (online)

\section{Preterm births a global problem}

Innovative solutions are needed to address the increasing incidence of preterm birth, according to a study reporting preterm birth rates for 184 countries. In 2010, 11.1\% of all live births worldwide were preterm. Of those 14.9 billion preterm babies, $60 \%$ were born in South Asia and subSaharan Africa. Higher preterm birth rates also occur in developed countries, with the US included in the list of the 10 countries with the highest number of preterm births. An editorial said the study was an urgent reminder of the importance of acting on preterm births as a major global public health issue, noting that "Worldwide investment in maternal health and pregnancy will reduce suffering and probably save life years at a scale never before seen in the history of public health".

Lancet 2012; 379: 2162-2172

Lancet 2012; 379: 2128-2129

\section{Herpesvirus linked to epilepsy}

The human herpesvirus (HHV) subtypes HHV-6B and HHV-7, commonly known as the roseola virus, account for around one-third of febrile status epilepticus (FSE), a US study has found. Febrile seizures occur in $2 \%-5 \%$ of children under 5 years. Around $5 \%-8 \%$ of cases meet the criteria for FSE, which is associated with a substantially increased risk of epilepsy, particularly temporal lobe epilepsy. The study, of 199 children aged 1 month to 5 years who presented with FSE, found HHV-6B in 54 children and HHV-7 in 12 children, two of whom also had HHV-6. The researchers will continue to follow the 199 children with FSE, of whom $30 \%-40 \%$ are expected to develop temporal lobe epilepsy.

Epilepsia 2012; 14 June (online) 